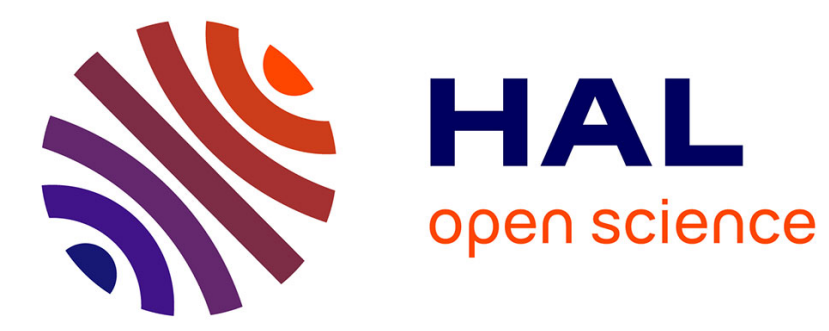

\title{
Molecular dynamics simulations for plasma-surface interactions
}

\author{
Erik Neyts, Pascal Brault
}

\section{To cite this version:}

Erik Neyts, Pascal Brault. Molecular dynamics simulations for plasma-surface interactions. Plasma Processes and Polymers, 2017, 14, pp.1600145. 10.1002/ppap.201600145 . hal-01358069

\section{HAL Id: hal-01358069 \\ https://hal.science/hal-01358069}

Submitted on 30 Aug 2016

HAL is a multi-disciplinary open access archive for the deposit and dissemination of scientific research documents, whether they are published or not. The documents may come from teaching and research institutions in France or abroad, or from public or private research centers.
L'archive ouverte pluridisciplinaire HAL, est destinée au dépôt et à la diffusion de documents scientifiques de niveau recherche, publiés ou non, émanant des établissements d'enseignement et de recherche français ou étrangers, des laboratoires publics ou privés. 


\title{
Molecular dynamics simulations for plasma-surface interactions
}

\author{
E. C. Neyts ${ }^{1}$, P. Brault ${ }^{2}$ \\ 1 University of Antwerp, Department of Chemistry, Research group PLASMANT, \\ Universiteitsplein 1, 2610 Wilrijk-Antwerp, Belgium \\ ${ }^{2}$ GREMI, UMR7344 CNRS Université d'Orléans BP6744, 45067 Orléans Cedex 2, France
}

Plasma-surface interactions are in general highly complex due to the interplay of many concurrent processes. Molecular dynamics simulations provide insight in some of these processes, subject to the accessible time and length scales, and the availability of suitable force fields. In this introductory tutorial-style review, we aim to describe the current capabilities and limitations of molecular dynamics simulations in this field, restricting ourselves to low-temperature non-thermal plasmas. Attention is paid to the simulation of the various fundamental processes occurring, including sputtering, etching, implantation and deposition, as well as to what extent the basic plasma components can be accounted for, including ground state and excited species, electric fields, ions, photons and electrons. A number of examples is provided, giving an bird's eye overview of the current state of the field.

Keywords: plasma-surface interactions, molecular dynamics, molecular simulation

\section{Introduction}

Molecular dynamics (MD) is a widely adopted computer simulation technique, tracing out the trajectories of all atoms in the system. As such, MD should be an invaluable simulation technique for investigating plasma-surface interactions, since it allows to study the dynamics of the system evolution with atomistic resolution.

Before adressing the application of MD to plasma-surface interactions, we here first provide a short historical background of MD simulations and how MD has become a scientific tool for studying plasma-surface interactions, followed by a short discussion on the fundamental assumptions of MD simulations. Subsequently, we also discuss the advantages and disadvantages of using either classical MD or ab-initio MD in the context of plasma-surface interactions.

In section 2, we shall address specific challenges in molecular dynamics related to studying plasma-surface interactions, in particular the plasma-specific factors that can or cannot be accounted for, and the often encountered disparity in time and length scales between simulations and experiments.

Then, in section 3, we describe how the various fundamental plasma-surface processes such as sputtering and etching are typically accounted for. Next, in section 4, we provide a large number of examples, focusing in each case on the value of MD simulation in the field considered, and what simulations have been performed so far. Finally, a summary and conclusion is given.

\subsection{Short historical background of MD simulations}

The first paper on molecular dynamics simulations is generally acknowledged to be a paper 
by Alder and Wainwright, studying the interactions in a system of hard spheres. ${ }^{[1]}$ Only three years later, a first paper came out relevant for plasma-surface interactions, by Gibson et al. on radiation damage in copper. ${ }^{[2]}$ The first MD simulation investigating a specific plasma process, sputtering, appeard in the late 1960s by the pioneering work of Harrison et al. on $\mathrm{Ar}^{+}$and $\mathrm{Xe}^{+}$sputtering of (again) copper. ${ }^{[3]}$ The potentials used in these and other papers on sputtering were two-body potentials. A two-body description of the forces is sufficient, as long as the kinetic energy transfer between the impinging particle and the surface is dominant, and the subsequent collision cascade is reminiscant of a series of binary collisions. Even in the study of sputtering, however, this approach has its limitations, as was explicitely acknowledged by, e.g., Garrison and coworkers. ${ }^{[4]}$ As soon as real chemical effects come into play, as is essential in describing, e.g., etching processes, a many-body potential is required. Such simulations became possible with the advent of the 3-body Stillinger-Weber potential for $\mathrm{Si}^{\left[{ }^{[5]}\right.}$ a few years later extended to include $\mathrm{Si}-\mathrm{F}$ interactions. ${ }^{[6]}$ Soon, the first simulations on Si-etching appeared. ${ }^{[7,8]}$ Around the same period, other many-body potentials for $\mathrm{Si}, \mathrm{C}$ and hydrocarbons were developed, paving the road for a multitude of MD simulations on these systems. ${ }^{[9,10,11]}$ An overview of some of the history of and potentials for MD simulations of plasma-surface interactions is provided in a review paper by Graves and Brault. ${ }^{[12]}$

\subsection{Fundamentals of $M D$ simulations}

Molecular dynamics simulations deterministically trace out the trajectory of all atoms in the system. Thus, any MD simulation essentially consists of solving suitable equations of motion using some suitable integration algorithm. ${ }^{[13,14,15]}$ Forces can be obtained either from a classical force field or from an electronic structure calculation, as is done in classical MD and ab initio MD, respectively. In section 1.3 , we will briefly confront both approaches.

In the large majority of MD simulations for plasma-surface interactions, however, classical simulations are employed, mainly due to the required number of atoms and the time scales involved in plasma processes. We shall here therefore restric ourselves to some details of classical simulations. In section 4 , however, we shall also point to some dedicated ab initio MD simulations for plasma-surface interactions.

Assuming a Hamiltonian system, the time evolution of the system is obtained by time integration of Newton's second law

$$
\mathbf{F}_{i}=-\nabla_{\mathbf{r}_{i}} V\left(\mathbf{r}_{1}(t), \ldots, \mathbf{r}_{N}(t)\right)=m_{i} \frac{\partial^{2} \mathbf{r}_{i}(t)}{\partial t^{2}}
$$

The force calculation is the most time consuming step in a MD simulation, and therefore an integration algorithm allowing for a large time step is preferential. Indeed, the time step should be chosen sufficiently small in order to resolve the fastest modes of motion and maintain a stable simulation. These modes are typically the vibrational motion of the atoms in a molecule. Typically, the time step is in the order of $0.1-2 \mathrm{fs}$. If a larger time step is used, the energy change will generally be too large, and hence the calculated forces will deviate from the actual forces. As a result, the total energy will not be conserved in the simulation (in an isolated system, i.e., in a microcanonical simulation) and the dynamics of the system will be erroneous.

In addition to conserving the total energy well and allowing for a large time step, the integration algorithm should also generate accurate trajectories, be time reversible, and be symplectic (i.e., conserve phase space density). Probably the most widely used integration 
algorithm obeying these requirements is the velocity-Verlet algorithm. ${ }^{[16]}$

A fundamental assumption of classical MD simulations is that the interactions between the atoms are represented well by the interatomic force field. Eventually, all classical MD simulations rely on the use of a sufficiently accurate interatomic force field. Indeed, if the potential energy function does not accurately reproduce the true potential energy function, the forces will in general also not be accurate, and hence all dynamics will be corrupted to at least some extent. Depending on the goal of the simulation, this condition of accuracy may be relaxed somewhat. If for instance one is only interested in equilibrium structures, then only the differences in the PES minima need to be accurate. If, on the other hand, one is interested in, e.g., pathways of chemical reactions, then also the entire path connecting any two minima must be represented correctly. We shall return to this issue in section 2.3.

It should be noted that the individual trajectories of atoms or individual events are generally not representative for the process. Indeed, MD is essentially a sampling technique, and conclusions should be based on statistically relevant data. Moreover, we typically require (or at least desire) the simulation to reproduce the essential physics and/or chemistry of the system, and reproduce trends, rather than exact values under one specific condition.

\subsection{Confronting classical and ab-initio MD simulations for plasma-surface interactions}

In classical MD simulations, forces propagating the atoms are calculated as the negative gradient of some suitable empirical interatomic force field (a.k.a. interatomic potential), describing the interactions between the atoms (see section 1.2). The potential energy landscape is thus taken to parametrically depend on the instantaneous positions of the atoms, and the electronic structure of the system only implicitely enters the description.

In ab initio MD simulations, on the other hand, the electronic structure of the system is actively taken into account. ${ }^{[17]}$ Several approaches are used. In Born-Oppenheimer MD (BOMD), the nuclei are considered classical particles which move according to the ionic forces which are calculated from the static electronic structure of the system. The electronic structure is solved in every step, given the current position of the nuclei. Thus, in every ionic step, the time-independent Schrödinger equation is solved, implying that the systems remains in the electronic ground state. In Ehrenfest $M D$, the nuclei are still propagated classically, but in contrast to BOMB, the electronic wavefunction is now explicitely solved for as a function of time, i.e., according to the time-dependent Schrödinger equation. Thus, Ehrenfest MD in principle allows to include non-adiabatic transitions between electronic states, within the framework of classically moving nuclei. In Car-Parrinello MD, finally, the electrons are explicitely included as active degrees of freedom. They follow fictitious dynamics keeping them close to the Born-Oppenheimer surface, i.e., maintaining the adiabaticity condition. Thus, as is the case in BOMD, electronic excitation cannot be modelled in this approach, but a siginificantly larger time step can be used, since an explicit electronic minimization is not needed.

In between classical and ab initio simulations, there is a variety of other methods which can be regarded as semi-empirical methods. These methods include e.g. the PM3 method and density functional based tight binding (DFTB) simulations, where some empirical or calculated parameters are used to significantly reduce the computational cost with respect to full ab initio methods, while still retaining access to the electronic structure.

Generally, the accuracy increases from classical force fields over semi-empirical methods to $a b$ initio methods, while the accessible time scale and number of atoms in the simulations 
increase in the opposite direction. As a result, the dynamical nature of plasma-surface interactions quickly renders the use of the more accurate methods infeasible. Indeed, even when a rather complex force field is used, such as e.g. the ReaxFF force field, the calculations are still orders of magnitude faster compared to, e.g., standard BOMD calculations.

\section{Challenges in molecular dynamics for plasma-surface interactions}

In principle, MD should be an ideal technique to study plasma-surface interactions at the atomic scale. The effective realization of such studies, however, is often hampered by two major issues. The first is that currently not all plasma components, including ground state and excited species, electromagnetic fields, charged species and photons, can (accurately) be accounted for. The second major issue is the discrepancy in time and length scales of what is practically feasible in terms of calculation time and the real time and length scales involved. As an introduction to the actual simulation of plasma-surface interactions, we shall start of with addressing the latter issue. Subsequently, we discuss the former issue, discussing how and to what extent one can (or cannot) simulate the various plasma components.

\subsection{Time scale issue and possible solutions}

In general, MD simulations typically do not exceed the nanosecond range, due to the requirements on the time step as mentioned in section 1.2. A time scale of a microsecond might still be feasible provided a sufficiently small system, a not too complex force field and sufficient patience. Anything beyond a microsecond, however, is practically excluded for most users, except for dedicated efforts. ${ }^{[18]}$

Various methods have been developed to go beyond this limitation. One class of solutions, so-called accelerated dynamics, ${ }^{[19]}$ is based on the concept of rare events. In this concept, the system is confined to the local potential energy surface (PES) minimum, until some rare thermal fluctuation allows it to escape the minimum and traverse a transition state into the next minimum. The rate constant is then given by the equilibrium flux through the dividing surface located at the saddle point separating the two minima. The idea of accelerated dynamics is to increase the rate of hopping from minimum to minimum. This can be accomplished by running several copies of the system on multiple processors (so-called parallel replica, ${ }^{[20]}$ ), running at high temperature and extrapolating to the (lower) temperature of interest (temperature accelerated dynamics, ${ }^{[21]}$ ) or by applying a boost potential, effectively lifting the minima of the PES while leaving the transition states untouched (hyperdynamics, ${ }^{[22]}$ ).

The latest development in this class, is so-called collective-variable driven hyperdynamics (CVHD), ${ }^{[23]}$ which is based on combining hyperdynamics with elements of metadynamics. ${ }^{[24]}$ CVHD is a fairly generic method capable of reaching time scales up to 100 's of seconds, thus increasing the time scale by up to a factor of $10^{9}$ relative to standard $M D,{ }^{[23,25]}$ while retaining its full atomistic detail. CVHD is currently the only accelerated dynamics technique applied to plasma-surface interactions. ${ }^{[26]}$

In CVHD, all degrees of freedom relevant for the processes to be observed are assumed to be projected onto a suitably chosen or constructed CV. Currently, CV's have been developed for bond breaking and for rotation around dihedral angles. ${ }^{[3]}$ Since in most surface processes bond breaking is indeed the rate limiting step, this CV will be sufficient in most cases. 
CVHD works by periodically adding a bias potential to the potential energy landscape of the current state of the system, as a function of the CV. A major advantage of CVHD is therefore that - as a method - it is not system dependent, and very little information on the process is required to be known in advance. Moreover, CVHD is self-learning in the sense that the bias is gradually grown, rather than a fixed value. This, in turn, enables CVHD to work on multiple, widely disparate time scales.

Because CVHD allows to extend the time scale by many orders of magnitude, it also allows to observe processes at temperatures much lower than is typically possible in regular MD, thus allowing to simulate processes at realistic temperatures. A further advantage of CVHD is that it only requires energies and forces as input, the source of which is irrelevant. Thus, CVHD may in principle be applied both to classical and ab-initio simulations.

The method also has two disadvantages. A first disadvantage is the need for an appropriate collective variable (CV). If the $\mathrm{CV}$ is not judiciously chosen, then relevant processes might be missed, thus corrupting the system evolution. If need be, however, CV's can be constructed and added relatively easily.

A second (minor) disadvantage of the method - relevant in particular for plasma-surface interactions - is the disparity in time scales between the impingment rate of species from the plasma and the rate of surface reactions. Indeed, CVHD only operates on processes describable by the chosen CV, and thus does not affect the impingment rate of species on the surface. As a result, the impingment rate (and hence the gas phase pressure) will still be much too high, as is the case in regular MD. In section 3.2.3., we elaborate on this issue in the context of plasma deposition studies. Note, however, that this can be easily circumvented by allowing only 1 gas phase particle in the simulation domain at a time in addition to the surface. Only after a suitable amount of time has passed, as dictated by the experimental pressure and as measured by CVHD, a new particle is added to the gas phase. In this way, deposition studies can be conducted on realistic time scales, at both realistic temperatures and pressures.

Another class of solutions is to couple MD with Monte Carlo simulations. ${ }^{[27,28]}$ This concept is build on the separation of time scales between reactive events and relaxation events. The reactive events typically take place on a ps - ns time scale, whereas relaxation occurs on longer time scales. In coupled or hybrid MD/MC simulations, reactive events are simulated in a MD module, while the system is relaxed by a subsequent $M C$ simulation. Note, however, that unless the time scale in the MC simulations is carefully calibrated, ${ }^{[29]}$ no actual time scale can be assigned to the hybrid $\mathrm{MD} / \mathrm{MC}$ simulation.

Finally, the effect of long time scales can also to a certain extent be incorporated through a stochastic description of part of the system. For instance, Abraham et al. proposed the use of adjustable diffusion coefficients, which can be set such that the ratio of the flux of particles to the surface in the simulation to the experimental particle flux equals the ratio of the set diffusion coefficient to the experimental diffusion coefficient. ${ }^{[30]}$ This approach was applied in the simulation of gold cluster growth in a sputter deposition setup.

\subsection{Length scale issue}

Besides the inherent difficulty of matching the experimental time scale to the simulation time scale, another problem often encountered is the mismatch in length scales. Consider for instance a typical plasma etching process in the microelectronics industry. The relevant surface features are in the order of tens or hundreds of nanometers. Say our system is a 
block of silicon with a volume of $100 \times 100 \times 10 \mathrm{~nm}^{3}$. This system then contains about $5 \times 10^{6}$ atoms. While this by itself can be constructed and simulated, it would be quite challenging to follow a system this size over a time scale of tens of $n s$ in order to simulate reactive events at the surface employing a sufficiently accurate and thus complex and time consuming force field. Nevertheless, the length scale issue is inherently easier to solve, since space can be easily partitioned is smaller blocks. Hence, simulations which are large in terms of the number of atoms they contain, can be easily parallellized.

\subsection{A few comments on interatomic potentials and MD codes}

As mentioned above in section 1.2., an essential requirement for (classical) MD simulations is that the interatomic force field used is sufficiently accurate for the system at hand. In particular in the case of plasma-surface interactions, a typical additional requirement is that the potential is a so-called reactive potential, allowing for chemical bonds to be broken and formed during the course of the simulation. This requirement is quite challenging, as the local environment of every atom should then be allowed to change as a function on time. Thus, specific features of reactive potentials as opposed to non-reactive potentials, which do not allow bonds to be broken or formed, is that all interactions should only depend on the element type and the environment, and that there is no pre-determined or fixed connectivity. Such potentials are generally based on the concept of a bond order, which is governed by the local environment of the atoms participating in the bond, and which in turn determines the energy of the bond. As a bond breaks, the bond order smoothly and continuously goes to zero, such that the total potential energy function remains differentiable at all points during the dissociation process. Examples of bond order potentials are the Brenner potentials, ${ }^{[11,31]}$ Tersoff potentials ${ }^{[9,10]}$ and ReaxFF. ${ }^{[32]}$

The number of potentials that are suitable for studying plasma-surface interactions is fairly limited. A number of potentials used in MD simulations of plasma/surface interactions is described in [12]. Often used potentials include the EAM potential for metals, ${ }^{[33]}$ and the above-mentioned bond-order potentials for (mostly) covalent materials, viz. the Brenner potential and potentials derived thereof for carbon-based systems, ${ }^{[11,31]}$ Tersoff potentials for silicon, ${ }^{[9]}$ and ReaxFF for a variety of systems. ${ }^{[32]}$

A multitude of MD codes exist. Well-known codes include the freely available LAMMPS code $^{[34]}$ and the Amsterdam Density Functional (ADF) code, ${ }^{[35]}$ which contain reactive potentials. LAMMPS is currently probably the most generic and versatile MD package available. ADF is very user friendly, in particular in view of its graphical user interface. It contains only the ReaxFF potential for reactive simulations. Other well-known codes include NAMD ${ }^{[36]}$ and Gromacs, ${ }^{[37]}$ which are however not reactive. NAMD and Gromacs are mostly used for biomolecules, often containing many millions of atoms.

\section{Addressing fundamental processes in plasma-surface interactions}

In this section we shall first shortly review typical plasma components, and how these may be accounted for in a MD simulation. Next, we shall describe how typical plasma processes can be simulated by MD.

\subsection{Plasma-specific components}

The different plasma-specific factors, distinguishing a plasma from a neutral gas, are summarized in Table 1. As indicated in the Table, some of these factors can be taken into account in MD simulations, while others cannot. Below, we describe the methods for accounting for these factors in some detail. 
Table 1 - Different plasma factors that can be accounted for in MD simulations. See text for explanation of the different factors.

\begin{tabular}{lll}
\hline Plasma factor & Possible? & Example \\
\hline electric field & yes & CNT growth \\
atoms and hyperthermal species & yes & Si-NW oxidation \\
radicals & yes & a-C:H growth \\
ions & yes & sputtering \\
electronically excited states & yes & electron etching \\
vibrationally excited states & yes / no & $/$ \\
photons & implicit & (polymer degradation) \\
electrons & yes & $/$ \\
\hline
\end{tabular}

\subsubsection{Ground-state neutrals: atoms, molecules and hyperthermal species}

Most MD simulations consider ground-state neutrals as the basic entities that build up the system. The fundamental building blocks may be either atoms, functional groups or entire molecules. In typical inorganic materials such as a metal, a sheet of graphene or a reactive molecule, one invariably works with atoms. In biomolecular simulations, however, which are often non-reactive, one typically uses a coarse-grained approach where each unit corresponds to a specific group of atoms, referred to as beads. For instance, instead of using 3 atoms to build the $-\mathrm{CH}_{2}-$ group, one uses a single bead representing this group. Groups may thus consist of only a few atoms, or may be larger units, depending on the needs of the simulation.

The reactivity of the atoms (or beads) is not accounted for by explicitely describing their electronic structure, but rather implictly by the precise functional shape of the interatomic potential. The energy of these atoms and beads is typically thermal. Thus, the initial velocities are often drawn from a Maxwellian distribution at the temperature of interest:

$$
f(\mathbf{v})=\left(\frac{m}{2 \pi k T}\right)^{3 / 2} \exp \left(\frac{-m \mathbf{v}^{2}}{2 k T}\right)
$$

It should be stressed here, however, that in particular cases it may be more appropriate to select velocities from a non-Maxwellian distribution function, as would e.g. be the case when considering sputtered atoms or ions in a plasma sheath. A recent example of this procedure is given in [38].

Hyperthermal species are species which have a kinetic energy significantly above thermal energy, in the order of a few eV. Such species can simply be represented by adding the required amount of kinetic energy to the particles. The main difference with low energy ions (see below), is that their translational motion is random, while the motion of the ions is usually governed to a good extent by the electric field.

\subsubsection{Excited neutrals}

While ground-state species are easily represented in classical MD, excited species are much more difficult. Indeed, the currently available interatomic potentials nearly always represent the ground-state of the system.

\subsection{3.a. Rotationally and vibrationally excited neutrals}

Provided that the temperature is not near or below the characteristic rotational temperature of the species involved, which is typically in the order of a few tens of $\mathrm{K}$, 
rotational excitation poses no specific difficulties in MD simulations. Indeed, the separation between rotational levels at temperatures relevant for typical plasma-surface processes is well below the thermal energy, and classical dynamics in combination with a continuum in the energy (instead of discrete levels) will in most cases reproduce rotational motion sufficiently accurate, since equipartition of energy is valid.

Vibrational excitation on the other hand is much more complicated. Indeed, the characteristic vibrational temperature is essentially always higher than the processing temperature of the plasma, and hence the separation between vibrational energy levels is large. MD simulations invariably employ classical dynamics to propagate the nuclei, and are therefore unable to account for this energy separation between levels. Consequently, the molecule may thus gain any vibrational energy - there is thus erroneously equipartition of energy implied also at low temperatures. A typical example of a property ill-represented is the heat capacity of molecules. Indeed, MD simulations will typically predict a heat capacity which is too high, since energy leaks into the vibrational modes.

When studying, e.g., the impact of molecules in their vibrational ground state on a surface at say room temperature, this is not so much of a problem, since the vibrational energy will in any case be low, and in most cases not significantly affect the surface process. However, it also prevents a quantitative or even qualitative correct treatment of vibrationally excited molecules impinging on a surface.

In spite of the neglect of the quantum nature of rotational and vibrational energy levels in $M D$, it is sometimes very useful to decouple the various energy terms in the energy analysis of the trajectories. Such an analysis allows for instance to investigate the mechanisms of translational and rotational energy transfer in cluster and molecule scattering from surfaces. ${ }^{[39,40,41]}$ Using semi-empirical molecular dynamics simulations, such energy transfer mechanisms have also been shown to be of importance in the plasma growth of hydrogenated silicon nanoparticles. ${ }^{[42]}$

\subsection{3.b. Electronically excited species}

$\mathrm{Ab}$ initio MD simulations where adiabaticity is not imposed are in principle capable of taking electronically excited species and the transition between electronic levels into account. In the case of classical simulations, taking electronic excitation into account seems quite impossible since interatomic potentials are invariably developed for species in their electronic ground state. Thus, in principle, one would need to develop an interatomic potential for each electronic state of the targeted species.

An elegant solution was presented by Su et al., by the introduction of the so-called eFF force field. ${ }^{[43,44]}$ In a conventional force field, the energy of the system is parameterized as a function of the nuclear coordinates. The parameterization is thus specific to the atom or bond modelled. Electrons are considered to reside in their ground state, and are not included explicitly. In eFF, in contrast, the electrons are explicitely accounted for, and modeled as Gaussian wave functions. The nuclei in turn are modelled as point charges. Thus, in eFF, there are no atom types or bond types, only electrons and nuclei. In efF, semiclassical equations of motion are integrated, following both the electrons and nuclei. A key point is that the electrons and nuclei can move independently from each other in eFF, such that the method allows for simulating electronically excited systems. The method was originally developed for elements with atomic number $Z=1-6$, and has been extended to cover up to the second and third row p-block elements. ${ }^{[4]]}$ So far, however, the method has to the best of our knowledge not yet been applied specifically to plasma-surface 
interactions.

Another very encouraging and recent development, so-called e-ReaxFF, is provided by the group of van Duin et al. ${ }^{[45]}$ In this method, electrons are again explicitly accounted for and modeled as Gaussian wave functions. The nuclei are treated as point charges. The advantage of e-ReaxFF is that it is developed in the framework of the existing ReaxFF scheme, which has been shown to be widely transferable and applicable to a very wide range of systems. So far, e-ReaxFF has been applied to the calculation of electron affinities of hydrocarbon molecules and electron transfer dynamics, and overall good agreement with ab initio calculations is demonstrated.

Alternatively, a much simpler but also very crude approximation might consist of locally modifying the term in the potential energy function governing the bond strength. Consider for instance a $\mathrm{C}_{2} \mathrm{H}_{4}$ molecule, which has 2 electrons in its $\pi_{\mathrm{cc}} \mathrm{HOMO}$ orbital. Exciting 1 electron to the $\pi^{*}$ cc LUMO orbital thus makes the bond order decrease from 2 to 1 . This can be modeled by adding a penalty energy term which is only activated for molecules to be excited. Clearly, however, such an approach is not suitable for modeling the difference in reactivity between, e.g., triplet oxygen and singlet oxygen.

\subsubsection{Ions}

Plasmas are characterized by the presence of charges, both positive and negative, in addition to neutral species. Sputtering, as induced most often by energetic ions, is frequently simulated. In classical molecular dynamics dealing with such simulations, ions are typically considered as fast neutrals, i.e., without charge. Practically, the "ion" is launched towards the substrate with a predefined incident energy and angle. The fact that a fast neutral is simulated instead of a real ion with a charge is usually justified by invoking Auger emission of an electron from the surface, which is captured by the ion before it actually hits the surface. While this is indeed an acceptable justification for metallic and semiconductor surfaces, this justification is not quite as clear for insulators. ${ }^{[46]}$ Note that eFF and e-ReaxFF described above can in principle also model ionic species.

\subsubsection{Electrons}

Although the effect of electrons can to a certain extent be simulated by standard molecular dynamics, i.e., the addition of energy or heat to a structure, inducing bond breaking etc., the electrons as such are not actually modeled, except for the recent developments of eFF and e-ReaxFF mentioned above. Often, electron impact on surfaces for typical low temperature plasmas is considered to be of little concern (with the exception perhaps of electron-induced secondary electron emission), and hence little attention is generally paid to electrons in MD simulations for plasmas. However, recent experiments pointed towards the active role of electrons in $\mathrm{C}-\mathrm{H}$ bond activation in plasma-catalytic dry reforming of methane on $\mathrm{Al}_{2} \mathrm{O}_{3^{-}}$ supported $\mathrm{Ni}$-catalysts. ${ }^{[47]}$

Moreover, plasma-generated electrons dissolving in a liquid have recently been shown to directly act as the reducing agent in an electrochemical setup ${ }^{[48]}$ Moreover, electrons are thought to play an important role in plasma medical applications as well. ${ }^{[49]}$ Thus, this topic is of significant current interest, and techniques for simulating these processes, in particular at the atomic level, would constitute a major advance.

\subsubsection{Electric fields}

External electric fields in MD are typically modeled by simply adding an extra one-body Lorentz force $\mathrm{F}=\mathrm{qE}$ to each charged atom. Thus, the atoms are accelerated or decelerated by 
the electric field, but the charges are not directly influenced by the electric field, nor is the electric field adapted to the charge distribution.

A more elaborate approach for simulating an electric field nearby metal surfaces was developed and applied by Djurabekova et al. ${ }^{[50]}$ In their approach, the MD model is complemented by a concurrent electrodynamics model. The electric field distribution over the metal surface (of arbitrary shape) is found by solving the Laplace equation on a threedimensional grid with a resolution similar to the size of a lattice atom. The Laplace equation is solved in every MD step, such that the time evolution of the electric field as a function of the instantaneous positions of the surface atoms is implicitely accounted for, since the positions of the surface atoms are updated in every MD step.

\subsubsection{Photons}

As is usually the case for electrons, also photons are not included directly in MD simulations. Again, however, their effect can be simulated, again by either by-artificially decreasing the bond order between a photon-irradiated bond (i.e., extracting potential energy), or alternatively by injecting an appropriate amount of kinetic energy into the structure. In practice, the latter method works by giving a kinetic energy, the recoil energy, to some randomly selected atom (the so-called recoil atom) in the structure. The direction of the velocity of the recoil atom is also selected randomly. An example of this approach is provided by Polvi et al. ${ }^{[51]}$ In principle, however, the anisotropy of the surface in principle prevents the latter method to be applied for studying electron- or phonon-induced damage at the surface.

\subsection{MD for plasma-specific processes}

Plasmas are highly versatile tools when it comes to modifying surfaces. By virtue of the above mentioned factors (neutrals, charged species, photons and fields), material may be sputtered from the surface, etched from the surface, deposited on the surface, or implanted in the surface. These basic processes in turn lead to numerous global surface modifications, including surface roughening, overall change in work function, change in adsorption capacity and catalytic activity, and so forth. Here, we will describe the four basic plasma-specific processes. Note again that we limit ourselves to low temperature non-equilibrium plasmas, thus excluding, e.g., plasma welding or plasma fusion processes.

\subsubsection{Sputtering}

Sputtering is probably the easiest plasma-surface process to model using molecular dynamics, and it was in fact the first typical process occuring in plasmas to be simulated by $M D .^{[3]}$ Although it is conceptually simple enough to simulate, some care must be taken in treating the energy transfer and ion accumulation in the target. ${ }^{[38]}$ In practice, an ion is positioned at a distance larger than the cutoff distance above the surface, and given a velocity corresponding to the required ion energy. Typically, the ion is directed normal to the surface, thus mimicking the typical electric field driven motion of the ion. The ion energy and momentum is transferred to the surface, which induces a collision cascade. Proper description of this process requires a relevant treatment of the energy dissipation of the incoming ion energy inside the target. This therefore requires to consider a relatively thick target model. This process eventually further leads to the ejection of atoms or clusters from the surface. Care should be taken in the choice of the interatomic potential as well. Indeed, the sputter process and sputter yield will naturally depend on the forces acting on the atoms, and thus on the steepness of the potential energy function. Some interatomic potentials, such as e.g. the Brenner potential, ${ }^{[11]}$ do not feature a sufficiently repulsive potential. In such case, splining the potential to a more repulsive potential for short 
atomic distances, such as a Molière potential or a ZBL-potential, should be considered.

\subsubsection{Etching}

Whereas sputtering is essentially a physical phenomenon, etching is much more of a chemical process. A plasma species arrives at the surface with (typically) thermal energy, reacts at the surface and thereby forms a volatile species. This volatile species then desorbs into the plasma again. In a MD simulation, this is simulated by allowing a thermalized particle, with a velocity drawn from a Maxwell-distribution at the appropriate temperature, to impinge on the surface at a random angle. Whereas in the case of (physical) sputtering in particular the repulsive wall of the interatomic potential is of importance, in the case of etching the attractive part of the potential is highly important. It turns out that taking manybody effects into account is essential to describe this process adequately. Consequently, it was only in the early 1990s, after the development of the first many-body potentials, that the first etching simulations appeared. ${ }^{[7,8]}$ Indeed, the precise shape of the PES determines the bond strengths in the equilibrium structure, and the stability of the transition state. Bonds must be broken (and formed) for etching to occur, and hence the associated energy barrier exponentially affects the corresponding rate constants. Moreover, if multiple reactions may occur with similar barriers, the accuracy of the product distribution will be governed by the accuracy of the potential function.

\subsubsection{Deposition and growth}

Deposition and growth studies are omnipresent in molecular dynamics. There are three main issues to consider. First, the time scale of deposition and growth is experimentally invariably many orders of magnitude slower that the slowest growth rate attainable in (regular) MD simulations. This can, at least in part, be avoided by application of accelerated MD techniques, as described above. Second, growth processes are seldom uniform over large areas down to the atomic level. Consequently, most, if not all, MD-based growth simulations use a simulation cell which is insufficiently large to represent the actual surface roughness and may not accurately sample the actual surface morphology. Third, neutral, non-radical growth species react only slowly at the surface in terms of the time scale attainable in MD. This problem is of course much less of an issue in plasma-based growth, since in many cases the radicals - which are very reactive at the surface - will often constitute the main growth species.

In practice, the surface is bombarded by a large number of sequentially impinging particles. The structure as resulting from each impact is used as the input configuration for the next impact. Thus, after several hundreds to thousands of impacts, a thin film develops. In view of the small lateral dimensions of the MD simulation cell, typically column-like structures are formed, which can be regarded as a sample cut from the thin film.

An important issue in such simulations is the disparity in the scale of the simulated time and the experimental time scale. Indeed, regular MD growth simulations typically inject a new particle every $1-20$ ps. Following the ideal gas law, this impingment flux is proportional to the gas pressure in the simulations:

$$
\Phi=\frac{p}{\sqrt{2 \pi m k T}}
$$

Thus, for an impingment flux $\Phi=1-0.05$ particles. $\mathrm{ps}^{-1} \cdot \mathrm{nm}^{-2}$ at a temperature of $300 \mathrm{~K}$, the corresponding pressure range of, say, a gas of methane, is $260-13 \mathrm{~atm}$. To reach a pressure of $1 \mathrm{~atm}$ for this gas and temperature, the time between impacts should be increased to 
about 250 ps. Considering that several hundreds to thousands of impacts are needed for a film to develop, this would amount to a total integration time of the order of $0.1-1 \mu \mathrm{s}$, even assuming a sticking coefficient close to 1 . Clearly, this is beyond what is typically attainable in a reasonable amount of time.

The direct result of this pressure problem (or, equivalently, impingment flux problem), is that all processes in between two impacts occuring on time scales beyond the ps range, are ignored. Depending on the system, this may include both local processes such as thermal diffusion of particles on the surface as well as global processes such as film relaxation and restructuring. Such long time scale processes may be covered by accelerated techniques such as CVHD or TAD, as mentioned above.

Another consequence of the pressure issue is that the carrier gas is typically ignored as well in the simulation, in view of the much lower concentration of growth species compared to the carrier gas. This approximation is justified is so far as the only role of the carrier gas is to thermalize the gas molecules and, in part, the surface, since thermalization in MD is typically accounted for by applying a suitable heat bath.

\subsubsection{Penetration and implantation}

When the impinging particles are ions or fast neutrals, these particles may penetrate and implant in the surface. This can be modelled relatively easy by regular MD, provided that the employed force field is sufficiently accurate, in particular with respect to its repulsive wall. Indeed, the nuclear stopping of the impinging species, and thus the penetration depth, is almost exclusively determined by the steepness of the repulsive wall of the interatomic potential. A second issue, somewhat particular to implantation studies, is to take heat dissipation into account. If the impinging particle is to implant in the structure, it needs to have sufficient kinetic energy. This energy will be transformed into heat through successive collisions, which in reality would be dissipated by the excited phonons in the solid. In a typical MD simulation, however, the boundaries in the lateral dimension are periodic, such that the heat cannot leave the system. Thus, a heat bath which is coupled to the system is applied. The strength of the heat bath and thus its coupling is typically controlled by some relaxation parameter. Thus, this parameter needs to be adjusted carefully in order to on the one hand avoid too strong a damping of the lattice vibrations and suppressing the occurrence of a thermal heat spike (in the case of very strong coupling), and on the other hand to avoid inducing artificial damage, reactions or other processes due to the nondissipation of the heat (in the case of very weak coupling). An often applied solution is to only apply a heat bath near the borders of the simulation cell, leaving the atoms in the $\{x, y\}$ center of the cell untouched.

\section{Selected applications}

In this section we provide examples of a number of applications of molecular dynamics to plasma-surface interactions, both from our own groups and from other groups. This overview is necessarily fragmented and non-exhaustive, but and is intended to provide a bird's eye view on the field. As mentioned before, we here restrict ourselves to lowtemperature non-equilibrium plasmas, and we shall not elaborate on the many fusion plasma related studies. Below, we present selected examples in the fields of thin film deposition, plasma sputtering, plasma oxidation, the calculation of sticking coefficients, plasma medicine, CNT growth and irradiation, plasma catalysis, cluster formation, and plasma etching.

\subsection{Plasma sputtering}


Plasma sputtering of a target is a process which is atomic in nature, making it an ideal topic of study for MD simulations. Indeed, traditionally, Traditionally, however, plasma sputtering is studied by either analytical models or by Monte Carlo (MC) simulations. Noteworthy in the analytical models is the pioneering work of Sigmund ${ }^{[52]}$ and refinements thereof, ${ }^{[53,54]}$ both on the collision cascade modelling and on the local target surface effects (e.g. roughness). As for the Monte Carlo simulations, both static (e.g., TRIM) and dynamical (e.g., TRYDYN and Dynam0) Monte-Carlo methods have been developed in the framework of the binary collision approximation. ${ }^{[55]}$ This latter approximation, however, excludes many-body contributions. Moreover accumulation of incoming ions in the substrate is not treated in this models.

To improve upon both the analytical and MC models, MD simulations may be applied, and MD simulations have for many year been quite popular to simulate plasma sputtering indeed. As always, MD simulations for sputtering require a suitable interatomic potential is to be available. The development of many-body potentials both for metal and semiconducting target materials is thus of major interest in this field.

An issue somewhat specific to MD simulations of plasma sputtering is to know the energy distribution of the sputtered atoms. Indeed, at low plasma pressure, the distribution is typically not Maxwellian due to the small collision number. Recently, the sputtered atom energy distribution was compared with available standard models. ${ }^{[38]}$ Future work in the field should consider sputtering of oxide target and reactive sputtering. Variable charge potentials as $\mathrm{COMB}^{[56]}{ }^{\text {reaxFF, }}{ }^{[57]}$ or modified Streitz-Mintmire ${ }^{[58]}$ will certainly lead to progress in the field.

\subsection{Thin film deposition: sputter deposition of metallic films}

Plasma sputter deposition may either refer to atom deposition resulting in atom clustering followed by film growth, ${ }^{[59,60]}$ or by direct deposition of clusters already formed in the plasma during transport from target to substrate ${ }^{[61]}$ An example is shown in Figure 1. A topic of current interest is the study of metallic film growth using MD simulations in view of the broad range of applications, including, e.g., the formation of complex alloys as high entropy alloys or metallic glass films which have unique properties, and nanocatalysts for which the field of applications is hugely broad. ${ }^{[62]}$ In the next section we shall consider the formation of silicon clusters in the plasma phase and deposition of thin silicon films. 

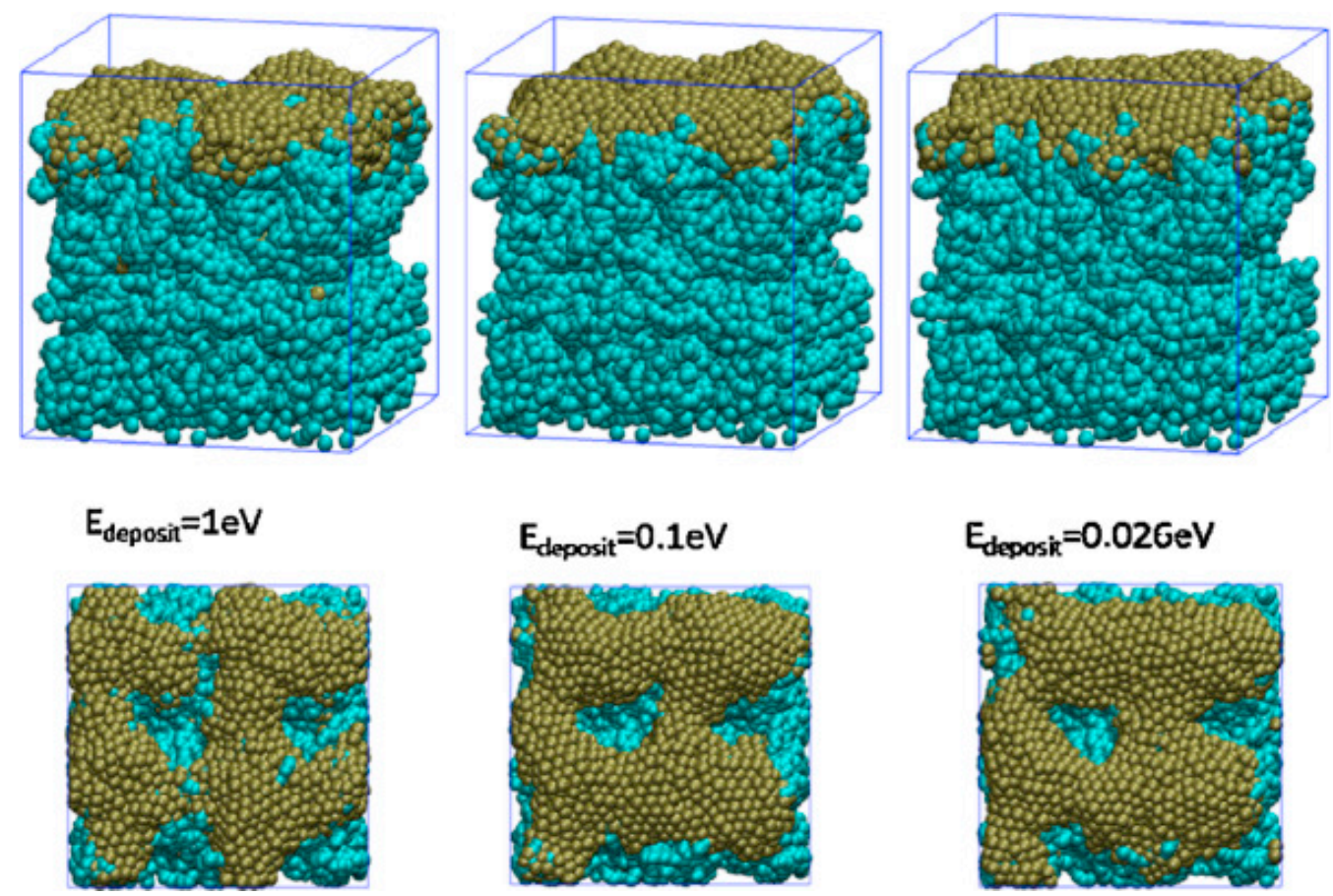

Figure 1 - Snapshots of Pt deposition on the porous carbon substrate. The green spheres represent $\mathrm{C}$-atoms and the brownish spheres represent Pt-atoms. Reproduced from [60] with permission from Elsevier.

As mentioned above, the correct treatment of the deposition process requires to know the kinetic energy / velocity distribution at the substrate. Obtaining the modified sputtered atom energy distribution escaping the target at the substrate location is therefore mandatory. For metal atom deposition the availability of many-body potentials such as Embedded Atom Method potentials has allowed considerable progress, since these potentials are typically well parametrized against both bulk and surface properties. ${ }^{[63]}$ Using MD simulations, many properties of cluster assemblies and films can be determined, including simulated $\mathrm{X}$-ray diffraction, radial distribution functions, energy barriers, surface energies, and annealing properties. This in turn allows direct comparison with experiments, except for the real time evolution. Indeed, as described above, the MD-generated fluxes are invariably unphysically large, and this is typically compensated for by ad hoc procedures. One approach to minimize this problem is to release the depositing atom at a rate which is sufficiently slow to allow for energy dissipation in the substrate. Increasing the time between releasing two atoms will then not directly affect the dynamics. Energy relaxation through phonons typically takes place in the picosecond time scale, so injecting atoms every 1 to 20 ps allows running MD simulations of sputter deposition on a reasonable time scale using parallel computing. ${ }^{[59]}$ However, long time scale processes, including thermal driven events such as thin film restructuring cannot be accounted for in these simulations. As mentioned above, novel methods such as CVHD may address this issue at a more fundamental level.

\subsection{Thin film deposition: PECVD of a-C:H}

Thin films of amorphous (hydrogenated) carbon (a-C(:H)) can have technologically important properties, such as high mechanical hardness, optical transparancy and chemical inertness. The typical application of $\mathrm{a}-\mathrm{C}(\mathrm{:H})$ is its use as a protective coating, e.g. for biomedical implants, on magnetic storage discs and optical windows. ${ }^{[64]}$ Depending on the exact 
structure, and in particular the $\mathrm{sp}^{3}: \mathrm{sp}^{2}: \mathrm{H}$ ratio, different types of amorphous carbon are distinguished, including glassy carbon, hard tetrahedral amorphous carbon (ta- $\mathrm{C}$ ) and softer hydrogenated amorphous carbon, each with their own specific applications. It is thus of interest to investigate how the growth process influences the resulting film structure and film properties. A recent review on MD simulations for plasma-surface interactions including ta- $\mathrm{C}$ thin film growth is given by Graves and Brault. ${ }^{[12]}$ We here focus on MD simulation of a$\mathrm{C}: \mathrm{H}$ thin films.

Although many MD studies have been performed on a-C and a-C:H film growth, only few of these relate directly to plasma-based growth. One set of such simulations mimicking PECVD set out to model a-C:H films as grown in an expanding thermal plasma (ETP). ${ }^{[65]}$ One of the characteristic features of the ETP is that it allows to growth $\mathrm{a}-\mathrm{C}: \mathrm{H}$ films at a very high rate (up to $70 \mathrm{~nm} / \mathrm{s},{ }^{[6]}$ ) with good quality and without applying a bias to the substrate. Thus, the growth proceeds in a purely chemical way, presumeably from the plasma-generated radicals. Experimentally, the fluxes of the various radicals may be tuned by varying the feedstock hydrocarbon gas and the carrier gas flux. ${ }^{[66]}$ Using MD simulations, relative good agreement in structure with the experimental films could be obtained. ${ }^{[67,68]}$ In particular, the MD simulations revealed the important role of the hydrogen flux in restructuring the film, demonstrating that the hydrogen flux may be used as an additional means of optimizing the film structure. ${ }^{[69]}$

Inevitably, however, these films always had a relatively low density, up to about $2 \mathrm{~g} / \mathrm{cm}^{3}$. In order to obtain denser and harder films, ion bombardment is required. Simulations of ionassisted a-C and a-C:H growth were provided by Marks, Belov et al. and Eckert et al. Marks, and Belov et al. performed a number of simulations where the growth species were energetic carbon ions. ${ }^{[70,71]}$ These simulations therefore correspond more to ion-beam growth than to PECVD. In the simulations of Eckert et al., the growth of the film was modelled by allowing thermal carbon species to impinge consecutively on the substrate, but additionally, a flux of energetic Ar ions was mixed in. ${ }^{[72]}$ These Ar-ions then resulted in a densification of the film. These simulations provide insight in how the film restructuring and densification is a function of the Ar-ion energy and Ar-ion flux.

\subsection{Plasma growth of silicon clusters and PECVD of thin silicon films}

Molecular dynamics simulations have frequently been used to study the atomic scale mechanisms of Si thin film formation, e.g., by cluster deposition, ${ }^{[73]}$ from sputtered $\mathrm{Si}^{\left[{ }^{[74]}\right.}$ from silane plasmas, ${ }^{[75]}$ and from specific silane-derived radicals. ${ }^{[76,77]}$ For instance, Sriraman et al. demonstrated through regular MD simulations the mechanism of hydrogen-induced crystallization of amorphous silicon films, as is relevant for a post-deposition treatment of the film by a hydrogen plasma. ${ }^{[78]}$ In particular, it was found that $\mathrm{H}$-atoms insert in strained $\mathrm{Si}-\mathrm{Si}$ bonds, thereby mediating the disorder-to-order transition. Such simulations demonstrate the capacity of MD simulations to provide a fundamental insight in operative mechanisms. The growth of amorphous hydrogenated $\mathrm{Si}$ thin films itself was also simulated by Sriraman et al, using $\mathrm{SiH}_{2}$ as a radical growth precursor. ${ }^{[76]}$ A number of characteristic film features was put forward, in particular a columnar structure. It is, however, not clear to what extent such features are the result of the neglect of long time scale dynamics, which are likely to affect the film structure and morphology during the growth process. Various other studies also addressed the growth of $\mathrm{Si}$ thin films from silane plasmas using MD simulations, both using classical potentials such as Stillinger-Weber or Tersoff-type potentials ${ }^{[77,79,75]}$ and using DFTB simulations. ${ }^{[80]}$ 
Another area of interest closely related to thin film growth is the formation of Si-clusters in the plasma phase. In contrast to surface processes, however, correctly treating energy dissipation during bond formation requires some care. In this case, explicitely accounting for the carrier gas, cooling the clusters as they are formed, may be considered.

Another issue in such calculations is the collision frequency. Indeed, the typical distance between atoms and molecules in the plasma (or gas) are far too large for a direct MD simulations. This issue may be circumvented by requiring that the collision number $\mathrm{n}_{\text {coll }}$ in the MD simulation box is smaller that the collision number encountered in the reaction zone in the experiment. The reaction number is given by $n_{\text {coll }}=P . d . \sigma / k_{b} T$, where $P$ is the pressure, $d$ the size and $T$ the temperature. Both in experiments and in simulation $T$ and $\sigma$ are the same. But as discussed above, $P$ is inevitably larger in MD simulations while the box size is much smaller than the reaction zone in the experiments. In principle, the MD collision number should not be larger than in the experiment in order to prevent forced reactivity. Once these issues are accounted for, cluster properties such as structure, composition, size distribution etc. are readily obtained.

Si clusters as formed in the plasma phase can be used as growth precursors for Si film growth. In this case, the film structure and characteristics will depend (at least in part) on the structure of the clusters. Gaining insight in the formation process of such clusters is therefore also of immediate importance for better understanding the film growth.

Vach et al. first employed fluid simulations to characterize the silane plasma, ${ }^{[81]}$ yielding values for the (relative) densities of the plasma radicals, their temperatures and their collision frequencies. These data were subsequently used as input for semi-empirical MD simulations at the PM3 level. These simulations allowed to follow the $\mathrm{Si}_{n} \mathrm{H}_{\mathrm{m}}$ cluster formation step by step, through successive additions of $\mathrm{SiH}_{4}$ molecules. It was found that depending on the energy of the impinging molecules on the growing clusters, the generated clusters were either amorphous or crystalline. In a more recent study, Le et al. used ab initio $M D$ to investigate $H$-induced heating and melting of such plasma-generated $\mathrm{Si}_{n} \mathrm{H}_{\mathrm{m}}$ nanoclusters. ${ }^{[82]}$ Thus, by tailoring the plasma conditions, the structure of the formed silicon clusters can be controlled, and therefore in part also the resulting thin film structure.

Note that also the formation of metal clusters may be simulated using MD simulations. Examples of such studies include the formation of iron and platinum nanoparticles. ${ }^{[83,84,85]}$

\subsection{Plasma Oxidation: $\mathrm{a}_{-} \mathrm{SiO}_{2}$}

At present, essentially the entire microelectronics field is based on silicon and silicon dioxide. Atomic scale control over the $\mathrm{Si} / \mathrm{SiO}_{2}$ interface, and the occurrence of the various suboxide species, is of great importance for applications such as MOSFETs, in particular in view of the ever decreasing device sizes. As an alternative to traditional thermal oxidation at elevated temperatures, typically above $1000 \mathrm{~K}$, hyperthermal oxidation at low temperature is envisaged as an alternative allowing for improved control over the oxide thickness and interface structure.

A number of MD simulations based on the ReaxFF force field have been performed in this field to elucidate how the oxidation processes proceeds as a function of the hyperthermal energy of the oxidizing species, the nature of the species, the shape of the substrate, and the temperature of the substrate. In the case of planar surfaces, it was found that the oxidation mechanism at low temperature differs from the mechanism at high temperature ${ }^{[86]}$ At low temperature, a seizable interface of $\mathrm{SiO}_{\mathrm{x}}$ species is found in the range 
6 - $15 \AA$, along with the build-up of an interfacial stress of about $2 \mathrm{GPa}^{[87]}$ At high temperature, the interface region is more extended, with a thickness of up to $35 \AA .{ }^{[8]}$

Similar studies were also carried out on Si-nanowires. Crystal core - amorphous shell Si/a$\mathrm{SiO}_{x}$ nanowires find applications in e.g. field-effect transistors and chemical sensors. Their electronic properties are strongly dependent on the precise nature of the interface region. From MD simulations, it was found that control over the core/shell structure is possible in ultra small nanowires at low temperature, due to a stress-induced self-limiting oxidation process. ${ }^{[89]}$ An example is shown in Figure 2 . The transition from complete oxidation of the nanowire to a core/shell structure was found to depend, on the one hand, on the combination of oxidation temperature and nanowire diameter, ${ }^{\left[{ }^{90]}\right.}$ and on the other hand on the plasma oxidation species. ${ }^{[91]}$
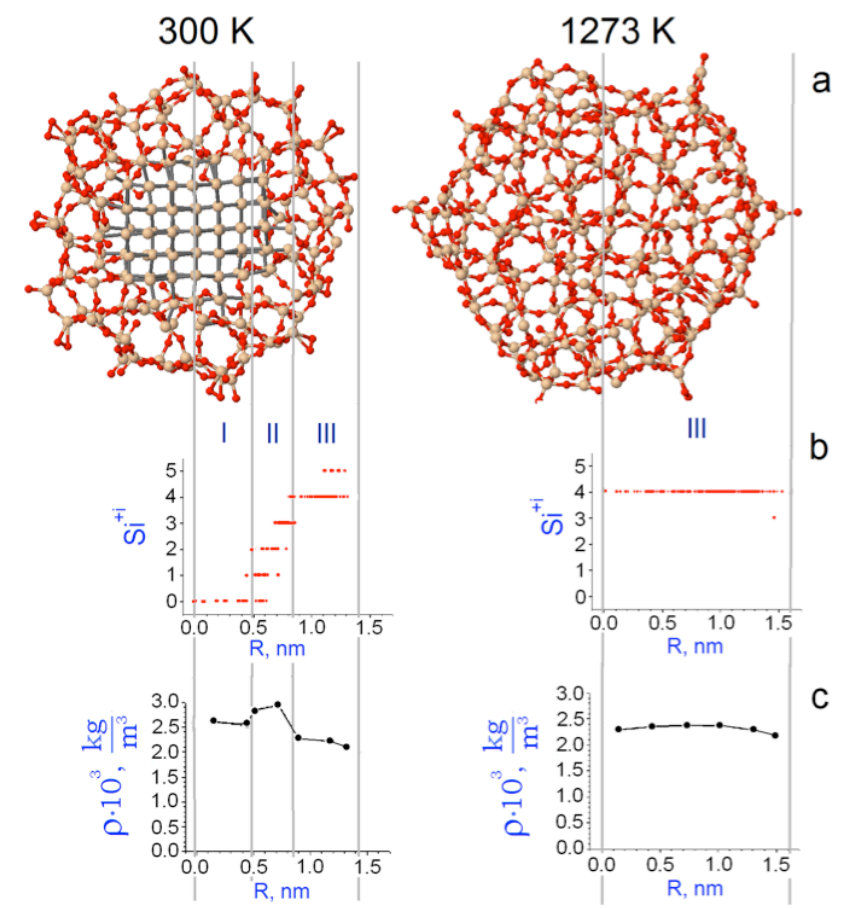

Figure 2 - Analysis of (a) post-oxidation Si-structures obtained at $300 \mathrm{~K}$ and $1273 \mathrm{~K}$ by (b) their Si-suboxide components and (c) their mass density distribution. Reproduced from [89] with permission from the American Chemical Society.

\subsection{Sticking coefficients}

A straightforward application of MD simulations is the calculation of sticking coefficient of plasma species on surfaces. This is of considerable importance, since such parameters are often used as input parameters in plasma models. Vice versa, plasma simulations often provide input data for MD simulations as well, such as the species reaching the surface, their fluxes and energies. An example of this approach was provided above in the context of plasma-growth of Si-clusters. ${ }^{[81]}$

Sticking coefficients may either be calculated for a surface which is considered to be representative for the experimental surface, or may be calculated for specific surface sites. Typically, quite a significant number of impacts is required in order to obtain statistically relevant results. This, in turn, makes the calculation of sticking coefficients of non-reactive species difficult. Indeed, suppose the sticking coefficient of some species to be in the order of $10^{-4}$, then on average only 1 impact out of 10000 attempts will result in a sticking event. If, for statistical reasons, we would wish to observe at least 50 sticking events, we need to 
perform at least $5 \times 10^{5}$ impacts. This is rather unpractical, in particular if many species of various surfaces are to be considered. Therefore, the literature so far mostly focusses on radical species such as hydrocarbon radicals or fluorine species, or highly reactive surfaces such as metal films.

Typical examples include the calculation of sticking coefficients of $\mathrm{CH}_{3}$ and other hydrocarbon species on diamond, relevant for diamond growth, ${ }^{[92,93,94,95]}$ amorphous carbon, relevant for a-C:H growth, and nickel surfaces, ${ }^{[67,96]}$ relevant for plasma catalysis, ${ }^{[97,98,99]}$ chlorine and fluorine species on Si-surfaces, relevant for plasma etching, ${ }^{[100,101]}$ oxygen species on Si-surfaces, relevant for Si-oxidation, ${ }^{[91,102]}$ as well as oxygen species on biomolecules, relevant for plasma medicine. ${ }^{[103]}$

\subsection{Plasma medicine}

Plasma medicine is one of the fields in non-thermal plasma science that is currently attracting a lot of attention. The goal is quite simple: can we improve existing therapies by using a plasma? Currently, succesful application is already reported in various fields, including on the direct plasma treatment of dermal wounds, dental treatment and bacterial desinfection. ${ }^{[104]}$ An important envisaged application is cancer treatment. Improving these applications obviously requires a thorough understanding of the underlying processes. So far, however, not much is known about the operative mechanisms in plasma medicine. This is complicated by the interplay of the many biochemical, chemical and physical phenomena taking place. Consequently, this is again a highly challenging topic for numerical simulation. Thus far, mostly non-atomistic simulations have been carried out. A recent review on simulations in general of plasma medicine can be found in [105], while a review specifically on atomistic simulations can be found in [106].

At the atomic scale, MD simulations relevant for plasma medicine were performed to study the interaction of plasma-generated radicals (reactive oxygen species, ROS) with various biomolecules, including the ROS-induced breakdown of peptidoglycan (as the basic component of the protective layer of gram-positive bacteria), relevant for bacterial desinfection, ${ }^{[107,108]}$ the interaction of ROS with DNA, relevant for cancer treatment, ${ }^{[109,110]}$ the interaction of ions and ROS with lipids, relevant for wounded skin treatment, ${ }^{[111,112,113]}$ and the interaction of radicals with liquids, relevant for the interaction of plasmas with biofilms. ${ }^{[114,103]}$ Another field where MD simulations have contributed to is the simulation of membrane electroporation, and the transport of molecules and ions through membrane channels. Examples include mechanistic studies on pore formation, ${ }^{[115,116]}$ ion transport though membrane pores, ${ }^{[117,118]}$ and membrane permeability as a function of the lipid peroxidation. ${ }^{[119]}$

However, in spite of the advance in our fundamental understanding of the processes mentioned above, it should be kept in mind that even a single cell is much larger than what can be simulated at the atomistic level. Also, it is important to realize that many of the relevant processes take place on the plasma/tissue level. Therefore, while atomistic simulation may bring a fundamental insight in the interactions between plasma species and individual molecules or small structures such as a cell membrane, they are not particularly suited for direct one-to-one comparison to experimental findings. Plasma medicine is inherently a highly complex theme, and a multi-level approach is deemed necessary.

\subsection{CNT growth}

Carbon nanotubes are technologically very interesting materials thanks to their extraordinairy mechanical and electronic properties, making them candidates for application 
as sensors, for hydrogen storage, transistor components, heat sinks and more. ${ }^{[120]}$ In particular their electronic and optical properties strongly depend on their exact geometric structure, i.e., on their chirality. Thus, as is the case for a-C:H thin films and $\mathrm{Si} / \mathrm{SiO}_{2}$ nanowires, their exact formation process determines their eventual properties and applications. The field of classical MD simulations for catalytic CNT growth was pioneered by the groups of Shibuta et al., ${ }^{[121,122]}$ Ding et al., ${ }^{[123,124]}$ and Balbuena et al.. ${ }^{[125,126]}$ In these works, and in fact in most subsequent works as well, ${ }^{[127]}$ it was invariably assumed that the hydrocarbon source molecules instantaneously decompose on the surface of the nanocatalyst. Thus, atomic carbon was used as the growth precursor. The carbon atoms dissolve into the cluster until supersaturation, and subsequently segregate at the surface. Further addition of carbon to the catalyst leads to the formation of a solid (single walled) carbon nanotube. Only recently, the first simulations yielding a specific chirality were reported. ${ }^{[128,129]}$ Also recently, the first MD simulations appeared where growth was accomplished from actual hydrocarbon molecules instead of starting from atomic carbon. ${ }^{[130]}$

With respect to plasma-enhanced catalytic growth of CNTs, much less work has been performed so far. As is the case for plasma catalysis, it is the combination of the required long time scales and the necessity to simultaneously take into account the various plasma factors that render such simulations highly challenging. Nevertheless, some progress was already made, in particular regarding the effect of adding an electric field, ${ }^{[131]}$ and the effect of low energy ion bombardment. ${ }^{[132,133,134]}$ MD simulations using the ReaxFF force field demonstrated that the effect of adding a static electric field was to gently drive the carbon atoms towards the tip of the nanocatalyst. Indeed, the electronegativity of the carbon atoms is (on the Pauling scale) 2.5, while the electronegativity of the nickel catalyst atoms is 1.9. Thus, the carbon atoms will be slightly negatively charged, and the electric field gradually pulls them towards the tip of the catalyst. This enhances the nucleation, and if a strong enough field was applied, vertically aligned CNTs emerged, in agreement with the experiment. ${ }^{[131]}$ In the case no electric field is applied, the CNTs grow in random directions.

The effect of ion bombardment during the growth of CNTs has been studied both experimentally and through simulations, see e.g. [135] and references therein. Employing ReaxFF-based MD simulations, the effect of low energy ion bombardment was studied by allowing Ar-ions with energies in the range $5-50 \mathrm{eV}$ to impinge on a partially formed CNT cap, covering a Ni-nanocatalyst. ${ }^{[132]}$ It was found that when the Ar-ions were given an energy in the range $10-25 \mathrm{eV}$, the nucleation is effectively enhanced. At lower energies, the Arions do not have sufficient energy to induce any significant changes in the carbon network. At energies above $25 \mathrm{eV}$, the Ar-ions have sufficient energy to effectively create defects and displace carbon atoms from the network, thus amorphizing the network. In the energy range $10-25 \mathrm{eV}$, however, the ions have just the right energy to break C-C bonds allowing a network restructuring, but do not have enough energy to actually induce new defects. Thus, the net effect in this energy range is the ion-induced defect healing in the carbon network. This is shown in Figure 3. Remarkably, the energy range as predicted by the MD simulations corresponds quantitatively with the experimentally determined range. ${ }^{[132]}$ 
a)

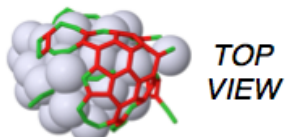

Ar bombardment
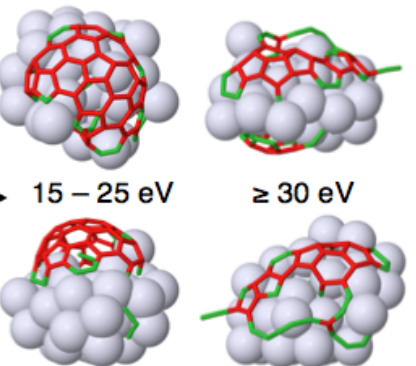

b)
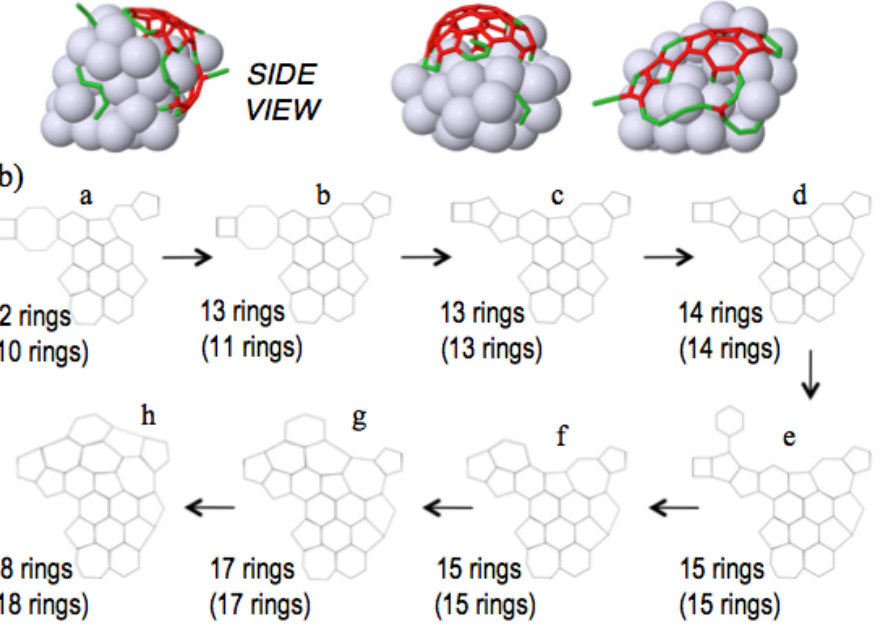

Figure $3-a)$ Observed defect healing and enhanced cap formation by ion bombardment in the energy range $15-25 \mathrm{eV}$ and destruction of the network at higher energies (> $30 \mathrm{eV}$ ). b) Growth of the carbon network due to the ion bombardment at $15 \mathrm{eV}$, as seen in the MD simulations. The numbers in parentheses indicate the sum of the pentagons, hexagons and heptagons; the other numbers indicate the total number of rings in the patch. Reproduced from [132] with permission from the American Physical Society.

Finally, also the plasma encapsulation of metal atoms in CNTs was studied using MD simulations. In particular, Kato et al. demonstrated the selective and damage-free encapsulation of Cs ions in CNTs, allowing the realization of pn-junction CNT thin films with excellent stability. Corresponding DFT calculations succeeded in explaining the experimentally observed optimal energy window for the $\mathrm{Cs}$ ions to penetrate the CNT. ${ }^{[136]}$

Plasma-based modification of closely related materials, such as metallofullerenes and graphene, have received less attention, at least from the simulation side. Nevertheless, some MD simulations have been performed, in particular on the plasma-based formation of metallofullerenes, ${ }^{[137,138]}$ similar to the above-mentioned Cs encapsulation in CNTs, and the plasma-growth of graphene under high flux conditions. ${ }^{[139]}$

\subsection{Plasma catalysis}

Plasma catalysis is one of the topics in the low-temperature plasma communtiy currently attracting significant interest. The basic idea is to improve the processing of a feedstock gas by combining the plasma with a catalyst. ${ }^{[140]}$ The goal can be either to convert a feedstock gas into a more valuable product, as is the case in dry reforming of $\mathrm{CH}_{4}$ with the formation of syngas or other value-added chemicals, the destruction of toxic or waste gases such as toluene, or the formation of an actual material, as is the case in plasma-catalytic growth of carbon nanotubes. The simulation of the latter process was described in section 4.8.

Molecular dynamics studies of plasma catalysis are complicated by the requirement to simultaneously take into account many of the typical plasma factors, including the presence of radicals, vibrationally and perhaps electronically excited species, ions, electrons, electric fields and perhaps even photons, all of which may contribute to the plasma catalytic 
process. ${ }^{[26]}$ Moreover, an additional complexity is the fact that plasma catalysis is very much a multi-element process, where the plasma species are typically composed of $2^{\text {nd }}$ row elements plus hydrogen, and the catalyst is typically a transition metal (or alloy thereof), possibly supported by a metal oxide. This combination thus requires a force field capable of accurately taking all interactions between the various atom types involved into account. Next to the standard issues of time and length scales, the combination of these two issues makes MD simulations of plasma catalysis highly challenging. A recent review on modelling plasma catalysis is given in [142].

So far, only very few attempts have been presentedin the literature, in particular focussing on the presence of radicals and atoms (as opposed to closed-shell molecules). Of current interest is MD simulations attempting to address hydrocarbon reforming reactions in the context of plasma catalysis. In this area, a number of MD studies appeared on the reactivity of $\mathrm{CH}_{\mathrm{x}}$ species on $\mathrm{Ni}$-surfaces for various temperatures in the context of dry reforming [66, $67,68] \cdot{ }^{[97,98,99]}$ Very recently, a CVHD simulation was also presented on the conversion of methanol to formaldehyde on a $\mathrm{V}_{2} \mathrm{O}_{5}$ surface, in the absence and presence of externally applied electric fields, as a first approximation to thermal catalysis and plasma catalysis, respectively. In this study, a time to first conversion in the range $0.1-100 \mathrm{~ms}$ was observed, which is clearly not attainable in regular MD simulations. ${ }^{[26]}$

\subsection{Plasma etching}

Plasma etching is probably one of the best well known applications of non-thermal plasmas. It is a process of crucial importance for the microelectronics industry. Research in this area is often directed towards improving properties of direct technological importance, such as the aspect ratio of etch channels, and tailoring the etch channel width. This ensures a continuous interest in the study of plasma etching, both through experiments and simulations. In fact, force fields are specifically developed for this application. ${ }^{[143]}$ As a result, there is a rich literature on MD-simulations of plasma etching, addressing topics such as the influence of the etchant gas, the energy of the plasma species, the role of concurrent ion bombardment, the surface temperature, etc. A typical example is given in [144], where MD simulations are applied to investigate the effect of $\mathrm{Cl}^{+}$and $\mathrm{Br}^{+}$ion impacts on the etching process of silicon in the presence of $\mathrm{Cl}$ and $\mathrm{Br}$.

Of significant current interest is crygenic etching. ${ }^{[145]}$ In this process, the wafer is kept at very low temperatures $\left(\sim-100{ }^{\circ} \mathrm{C}\right)$, in order to minimize diffusion of dopants or defects into the wafer. Recent MD simulations on fluorine etching of Si-wafers elucidated how the formation of a weakly bound physisorbed layer of $\mathrm{SiF}_{4}$ is responsible for the experimentally observed differences between thermal and cryogenic etching. ${ }^{[101]}$

\section{Summary and conclusions}

Molecular dynamics (MD) simulations are a highly versatile tool to investigate processes at the atomic scale, including surface processes. Thus, MD comes as a natural technique to investigate the interaction between non-equilibrium plasmas and surfaces as well. While a variety of simulations, for various applications, have indeed already been carried out, the study of plasma-surface interactions through MD simulations is hampered by essentially two problems: the specific plasma factors that can be accounted for (including atoms, molecules, radicals, excited species, photons, electrons, ions, and electric fields), and the attainable time and length scales. In the latter field, siginificant progress was recently made with the advent of new techniques addressing long-time scales. How to take into account some of the former issues, however, in particular photons, electrons and vibrationally excited molecules, currently remains an open question. The development of new techniques, such 
as CVHD, new (improved) force fields and their parametrizations, such as e-ReaxFF, in combination with the ever increasing computer speed, is likely to bring atomistic simulations closer to plasma-surface interaction experiments, while providing a fundamental and atombased insight in the underlying mechanisms.

\section{Keywords}

molecular dynamics

surfaces

interfaces

\section{References}

[1] G. J. Alder, T. E. Wainwright, J. Chem. Phys. 1957, 27, 1208

[2] J. B. Gibson, A. N. Goland, M. Milgram, G. H. Vineyard, Phys. Rev. 1960, 120, 1229

[3] D. E. Harrison Jr., N. S. Levy, J. P. Johnson III, H. M. Effron, J. Appl. Phys. 1968, 39, 3742

[4] B. J. Garrison, C. T. Reimann, N. Winograd, D. E. Harrison Jr., Phys. Rev. B 1987, 36, 3517

[5] F. H. Stillinger, T. A. Weber, Phys. Rev. B 1985, 31, 5262

[6] F. H. Stillinger, T. A. Weber, Phys. Rev. Lett. 1989, 62, 2144

[7] T. A. Schoolcraft, B. J. Garrison, J. Am. Chem. Soc. 1991, 113, 8221

[8] C. J. Wu, E. A. Carter, J. Am. Chem. Soc. 1991, 113, 9061

[9] J. Tersoff, Phys. Rev. B 1988, 37, 6991

[10] J. Tersoff, Phys. Rev. Lett. 1988, 61, 2879

[11] D. W. Brenner, Phys. Rev. B 1990, 42, 9458

[12] D. B. Graves, P. Brault, J. Phys. D: Appl. Phys. 2009, 42, 194011

[13] M. P. Allen, D. J. Tildesley, Computer simulation of liquids, Oxford University Press, Oxford, United Kingdom, 1987

[14] J. M. Haile, Molecular dynamics simulation, John Wiley \& Sons Inc., New York, USA, 1997

[15] D. Frenkel, B. Smit, Understanding molecular simulation, Academic Press, Elsevier, London, United Kingdom, 2002

[16] W. C. Swope, H. C. Andersen, P. H. Berens, K. R. Wilson, J. Chem. Phys. 1982, 76, 637

[17] D. Marx, J. Hutter, Ab initio molecular dynamics - basic theory and advanced methods, Cambridge University Press, 2009

[18] D E. Shaw et al., Proc. Conf. High Performance Computing Networking, 2009, DOI: 10.1145/1654059.1654099

[19] A. F. Voter, F. Montalenti, T. C. Germann, Annu. Rev. Mater. Res. 2002, 32, 321

[20] A. F. Voter, Phys. Rev. B 1998, 57, 13985

[21] M. R. Sørensen, A. F. Voter, J. Chem. Phys. 2000, 112, 9599

[22] A. F. Voter, J. Chem. Phys. 1997, 106, 4665

[23] K. M. Bal, E. C. Neyts, J. Chem. Theory Comput. 2015, 11, 4545

[24] A. Laio, M. Parrinello, PNAS 2002, 99, 12562

[25] K. M. Bal, E. C. Neyts, Chem. Sci. 2016, 7, 5280

[26] E. C. Neyts, submitted

[27] E. C. Neyts, A. Bogaerts, Theor. Chem. Acc. 2013, 132, 1320

[28] M. J. Mees, G. Pourtois, E. C. Neyts, B. J. Thijsse, A. Stesmans, Phys. Rev. B 2012, 85, 134301

[29] K. M. Bal, E. C. Neyts, J. Chem. Phys. 2014, 141, 204104

[30] J. W. Abraham, T. Strunskus, F. Faupel, M. Bonitz, J. Appl. Phys. 2016, 119, 185301

[31] S. J. Stuart, A. B. Tutein, J. A. Harrison, J. Chem. Phys. 2000, 112, 6472

[32] A. C. T. van Duin, S. Dasgupta, F. Lorant, W. A. Goddard, J. Phys. Chem. A 2001, 105, 9396

[33] M. S. Daw, M. I. Baskes, Phys. Rev. Lett. 1983, 50, 1285 
[34] S. Plimpton, J. Comp. Phys. 1995, 117, 1

[35] G. te Velde, F. M. Bickelhaupt, S. J. A. van Gisbergen, G. Fonseca Guerra, E. J. Baerends, J. G. Snijders, T. Ziegler, J. Comput. Chem. 2001, 22, 931

[36] J. C. Philips, R. Braun, W. Wang, J. Gumbart, E. Tajkhorshid, E. Villa, C. Chipot, R. D. Skeel, L. Kalé, K. Schulten, J. Comput. Chem. 2005, 26, 1781

[37] H. J. C. Berendsen, D. van der Spoel, R. van Drunen, Comp. Phys. Commun. 1995, 91, 43

[38] P. Brault, S. Chuon, J.-M. Bauchire, Frontiers in Physics 2016, 4, 20

[39] D. M. Koch, G. H. Peslherbe, H. Vach, J. Chem. Phys. 2001, 115, 7685

[40] H. Ambaye, J. R. Manson, J. Chem. Phys. 2006, 125, 084717

[41] T. N. V. Nguyen, D. M. Koch, G. H. Peslherbe, H. Vach, J. Chem. Phys. 2003, 119, 7451

[42] H. Vach, Q. Brulin, N. Chaâbane, T. Novikova, P. Roca i Cabarrocas, B. Kalache, K. Hassouni, S. Botti, L. Reining, Comput. Mater. Sci. 2006, 35, 216

[43] J. T. Su, W. A. Goddard, Phys. Rev. Lett. 2007, 99, 185003

[44] A. Jaramillo-Botero, J. T. Su, Q. An, W. A. Goddard, J. Comput. Chem. 2011, 32, 497

[45] M. M. Islam, G. Kolesov, T. Verstraelen, E. Kaxiras, A. C. T. van Duin, J. Chem. Theory Comput. 2016, DOI: 10.1021/acs.jctc.6b00432

[46] Hannspeter Winter, Joachim Burgdörfer, Eds., Slow Heavy-Particle Induced Electron Emission from Solid Surfaces, Springer-Verlag, Berlin Heidelberg, 2007, p. 104

[47] J. Kim, M. S. Abbott, D. B. Go, J. C. Hicks, ACS Energy Lett. 2016, 1, 94

[48] C. Richmonds, M. Witzke, B. Bartling, S. W. Lee, J. Wainright, C.-C. Liu, R. M. Sankaran, J. Am. Chem. Soc. 2011, 133, 17582

[49] J. Heinlin, G. Morfill, M. Landthaler, W. Stolz, G. Isbary, J. L. Zimmermann, T. Shimizu, S. Karrer, J. Dtsch. Dermatol. Ges. 2010, 8, 968

[50] F. Djurabekova, S. Parviainen, A. Pohjonen, K. Nordlund, Phys. Rev. E 2011, 83, 026704

[51] J. Polvi, K. Nordlund, Nucl. Instrum. Methods B 2013, 312, 54

[52] P. Sigmund, Phys. Rev. 1969, 184, 383

[53] Y. Yamamura, H.Tawara, At. Data Nucl. Data Tables 1996, 62, 149

[54] F. Boydens, W.P. Leroy, R. Persoons, D. Depla, Thin Solid Films 2013, 531, 32

[55] Ono T, Kenmotsu T, Muramoto T. Simulation of the sputtering process. In: D. Depla and S. Mahieu, Eds. Reactive Sputter Deposition. Berlin: Springer-Verlag, 2009, p. 1-42.

[56] J. Yu, S. B. Sinnott, and S. R. Phillpot, Phys Rev B 2007, 75, 085311

[57] Y. K. Shin, T-R Shan, T. Liang, M. J. Noordhoek, S. B. Sinnot, A. C. T. van Duin, S. R. Phillpot, MRS Bulletin 2012, 37, 504

[58] X. W. Zhou, H. N. G. Wadley, J.-S. Filhol, M. N. Neurock, Phys. Rev. B 2004, 69, 035402

[59] L. Xie, P. Brault, J.-M. Bauchire, A.-L. Thomann, L. Bedra, J. Phys D: Appl. Phys. 2014, 47, 224004

[60] L. Xie, P. Brault, C. Coutanceau, J.-M. Bauchire, A. Caillard, S. Baranton, J. Berndt, E. C. Neyts, Appl. Catal. B: Environ. 2015, 162, 21

[61] H. Haberland, Z. Insepov, M. Moseler, Phys. Rev. B 1995, 51, 11061

[62] P. Brault and E. Neyts, Catal. Today 2015, 256, 3

[63] S. Foiles, M. I. Baskes, MRS Bulletin 2012, 37, 485

[64] J. Robertson, Mater. Sci. Eng. 2002, R37, 129

[65] J. Benedikt, M. Wisse, R. V. Woen, R. Engeln, M. C. M. van de Sanden, J. Appl. Phys. 2003, 94, 6932

[66] J. Benedikt, D. J. Eijkman, W. Vandamme, S. Agarwal, M. C. M. van de Sanden, Chem. Phys. Lett. 2005, 402, 37

[67] E. Neyts, A. Bogaerts, R. Gijbels, J. Benedikt, M.C.M. van de Sanden, Diamond Relat. Mater. 2004, 13, 1873

[68] E. Neyts, A. Bogaerts, M. C. M. van de Sanden, J. Appl. Phys. 2006, 99, 014902

[69] E. Neyts, A. Bogaerts, M. C. M. van de Sanden, Appl. Phys. Lett. 2006, 88, 141922

[70] N. A. Marks, Diamond Relat. Mater. 2005, 14, 1223 
[71] H. U. Jäger, A. Yu. Belov, Phys. Rev. B 2003, 68, 024201

[72] E. Neyts, M. Eckert, A. Bogaerts, Chem. Vap. Depos. 2007, 13, 312

[73] J. Q. Xi, J. Y. Feng, H. W. Lu, Modelling Simul. Mater. Sci. Eng. 1999, 7, 289

[74] A.-P. Prskalo, S. Schmauder, C. Ziebert, J. Ye, S. Ulrich, Comput. Mater. Sci. 2011, 50, 1320

[75] M. Matuskama, S. Hamaguchi, Thin Solid Films, 2008, 516, 3443

[76] S. Sriraman, E. S. Aydil, D. Maroudas, J. Appl. Phys. 2004, 95, 1792

[77] T. Bakos, M. S. Valipa, D. Maroudas, J. Chem. Phys. 2007, 126, 114704

[78] S. Sriraman, S. Agarwal, E. S. Aydil, D. Maroudas, Nature, 2002, 418, 62

[79] T. Ohira, O. Ukai, M. Noda, Surf. Sci. 2000, 458, 216

[80] T. Kuwahara, H. Ito, K. Kawaguchi, Y. Higuchi, N. Ozawa, M. Kubo, Sci. Rep. 2015, 5, 9052

[81] H. Vach, Q. Brulin, C. Chaâbane, T. Novikova, P. Roca i Cabarrocas, B. Kalache, K. Hassouni, S. Botti, L. Reining, Comput. Mater. Sci. 2006, 35, 216

[82] H. L. T. Le, N. C. Forero-Martinez, H. Vach, Phys. Status Solidi A, 2014, 211, 294

[83] N. Lümmen, T. Kraska, Nanotechnology 2004, 15, 525

[84] N. Lümmen, T. Kraska, Nanotechnology 2005, 16, 2870

[85] N. Lümmen, T. Kraska, Eur. Phys. J. D, 2007, 41, 247

[86] U. Khalilov, G. Pourtois, S. Huygh, A. C. T. van Duin, E. C. Neyts, A. Bogaerts, J. Phys. Chem. C 2013, 117, 9819

[87] U. Khalilov, G. Pourtois, A. C. T. van Duin, E. C. Neyts, J. Phys. Chem. C 2012, 116, 21856

[88] U. Khalilov, G. Pourtois, A. C. T. van Duin, E. C. Neyts, J. Phys. Chem. C 2012, 116, 8649

[89] U. Khalilov, G. Pourtois, A. C. T. van Duin, E. C. Neyts, Chem. Mater. 2012, 24, 2141

[90] U. Khalilov, G. Pourtois, A. Bogaerts, A. C. T. van Duin, E. C. Neyts, Nanoscale 2013, 5, 719

[91] U. Khalilov, M. Yusupov, A. Bogaerts, E. C. Neyts, J. Phys. Chem. C 2016, 120, 472

[92] L. Schwaederle, P. Brault, C. Rond, A. Gicquel, Plasma Process. Polym. 2015, 12, 764

[93] P. Träskelin, E. Salonen, K. Nordlund, A. V. Krasheninnikov, J. Keinonen, C. H. Wu, J. Appl. Phys. 2003, 93, 1826

[94] M. Eckert, E. Neyts, A. Bogaerts, J. Phys. D.: Appl. Phys. 2008, 41, 032006

[95] M. Eckert, E. Neyts, A. Bogaerts, Chem. Vap. Depos. 2008, 14, 213

[96] E. Neyts, M. Tacq, A. Bogaerts, Diamond and Relat. Mater. 2006, 15, 1663

[97] W. Somers, A. Bogaerts, A.C.T. van Duin, E. Neyts, J. Phys. Chem. C. 2012, 116, 20958

[98] W. Somers, A. Bogaerts, A. C. T. van Duin, S. Huygh, K. M. Bal, E. C. Neyts, Catal. Today 2013, 211, 131

[99] W. Somers, A. Bogaerts, A. C. T. van Duin, E. C. Neyts, Appl. Catal. B: Environ. 2014, 154155,1

[100] F. Gou, E. Neyts, M. Eckert, S. Tinck and A. Bogaerts, J. Appl. Phys. 2010, 107, 1133005

[101] S. Tinck, E. C. Neyts, A. Bogaerts, J. Phys. Chem. C 2014, 118, 30315

[102] E. C. Neyts, U. Khalilov, G. Pourtois, A. C. T. van Duin, J. Phys. Chem. C 2011, 115, 4818

[103] N. Khosravian, A. Bogaerts, S. Huygh, M. Yusupov, E. C. Neyts, Biointerphases 2015, 10, 029501

[104] M. G. Kong, G. Kroesen, G. Morfill, T. Nosenko, T. Shimizu, J. van Dijk, J. L. Zimmermann, New J. Phys. 11, 2009, 115012

[105] E. C. Neyts, M. Yusupov, C. C. Verlackt, A. Bogaerts, J. Phys. D: Appl. Phys. 2014, 47, 293001

[106] A. Bogaerts, N. Khosravian, J. Van der Paal, C. C. W. Verlackt, M. Yusupov, B. Kamaraj, E. C. Neyts, J. Phys. D: Appl. Phys. 2016, 49, 054002

[107] M. Yusupov, E. C. Neyts, U. Khalilov, R. Snoeckx, A. C. T. van Duin, A. Bogaerts, New J. Phys. 2012, 14, 093043

[108] M. Yusupov, A. Bogaerts, S. Huygh, R. Snoeckx, A. C. T. van Duin, E. C. Neyts, J. Phys. Chem. C 2013, 117, 5993 
[109] R. M. Abolfath, A. C. T. van Duin, T. Brabec, J. Phys. Chem. B 2011, 115, 11045

[110] C. C. W. Verlackt, E. C. Neyts, T. Jacob, D. Fantauzzi, M. Golkaram, Y.-K. Shin, A. C. T. van Duin, A. Bogaerts, New J. Phys. 2015, 17, 103005

[111] N. Y. Babaeva, N. Ning, D. B. Graves, M. J. Kushner, J. Phys. D: Appl. Phys. 2012, 45 115203

[112] J. Van der Paal, S. Aernouts, A. C. T. van Duin, E. C. Neyts, A. Bogaerts, J. Phys. D: Appl. Phys. 2013, 46, 395201

[113] J. Van der Paal, C. C. Verlackt, M. Yusupov, E. C. Neyts, A. Bogaerts, J. Phys. D: Appl. Phys. 2015, 48, 155202

[114] M. Yusupov, E. C. Neyts, P. Simon, G. Berdiyorov, R. Snoeckx, A. C. T. van Duin, A. Bogaerts, J. Phys. D: Appl. Phys. 2014, 47, 025205

[115] D. P. Tieleman, H. Leontiadou, A. E. Mark, S.-J. Marrink, J. Am. Chem. Soc., 2003, 125, 6382

[116] Q. Hu, Z. Zhang, H. Qiu, M. G. Kong, R. P. Joshi, Phys. Rev. E, 2013, 87, 032704

[117] Q. Hu, S. Viswanadham, R. P. Joshi, K. H. Schoenbach, S. J. Beebe, P. F. Blackmore, Phys. Rev. E, 2005, 71, 031914

[118] A. Gurtovenko, I. Vattulainen, J. Am. Chem. Soc., 2005, 127, 17570

[119] J. Van der Paal, E. C. Neyts, C. C. W. Verlackt, A. Bogaerts, Chem. Sci. 2016, 7, 489

[120] M. F. L. De Volder, S. H. Tawfick, R. H. Baughman, A. John Hart, Science 2013, 339, 535

[121] Y. Shibuta, S. Maruyama, Chem. Phys. Lett. 2003, 382, 381

[122] Y. Shibuta, J A Elliott, Chem. Phys. Lett. 2006, 427, 365

[123] F. Ding, A. Rosen, K. Bolton, J. Chem. Phys. 2004, 121, 2775

[124] F. Ding, K. Bolton, A. Rosen, Appl. Surf. Sci. 2006, 252, 5254

[125] J. Zhao, A. Martinez-Limia, P. B. Balbuena, Nanotechnology 2005, 16, S575

[126] M. A. Ribas, F. Ding, P. B. Balbuena, B. I. Yakobson, J. Chem. Phys. 2009, 131, 224501

[127] J. A. Elliott, Y. Shibuta, H. Amara, C. Bichara, E. C. Neyts, Nanoscale 2013, 5, 6662

[128] E. C. Neyts, Y. Shibuta, A. C. T. van Duin, A. Bogaerts, ACS Nano 2010, 4, 6665

[129] E. C. Neyts, A. C. T. van Duin, A. Bogaerts, J. Am. Chem. Soc. 2011, 133, 17225

[130] U. Khalilov, A. Bogaerts, E. C. Neyts, Nat. Commun. 2015, 6, 10306

[131] E. C. Neyts, A. C. T. van Duin, A. Bogaerts, J. Am. Chem. Soc. 2012, 134, 1256

[132] E. C. Neyts, K. Ostrikov, Z. J. Han, S. Kumar, A. C. T. van Duin, A. Bogaerts, Phys. Rev. Lett. 2013, 110, 065501

[133] M. Shariat, B. Shokri, E. C. Neyts, Chem. Phys. Lett. 2013, 590, 131

[134] M. Shariat, S. I. Hosseini, B. Shokri, E. C. Neyts, Carbon 2013, 65, 269

[135] E. C. Neyts, Front. Chem. Sci. Eng. 2015, 9, 154

[136] T. Kato, E. C. Neyts, Y. Abiko, T. Akama, R. Hatakeyama, T. Kaneko, J. Phys. Chem. C 2015, 119, 11903

[137] E. Neyts, A. Bogaerts, Carbon 2009, 47, 1028

[138] E. Neyts, A. Maeyens, G. Pourtois, A. Bogaerts, Carbon 2011, 49, 1013

[139] E. C. Neyts, A. C. T. van Duin, A. Bogaerts, Nanoscale 2013, 5, 7250

[140] E. C. Neyts, K. Ostrikov, M. K. Sunkara, A. Bogaerts, Chem. Rev. 2015, 115, 13408

[141] E. C. Neyts, Plasma Chem. Plasma Processing 2016, 36, 185

[142] E C Neyts, A Bogaerts, J. Phys. D: Appl. Phys. 2014, 47, 224010

[143] H. Ohta, S. Hamaguchi, J. Chem. Phys. 2001, 115, 6679

[144] N. Nakazaki, Y. Takao, K. Eriguchi, K. Ono, J. Appl. Phys. 2015, 118, 233304

[145] R. Dussart, T. Tillocher, P. Lefaucheux, M. Boufnichel, J. Phys. D: Appl. Phys. 2014, 47, 123001 


\section{Summary / Table of contents text}

In this review, we describe the current capabilities and limitations of molecular dynamics simulations of plasma-surface interactions. We pay specific attention to the simulation of the various fundamental processes, as well as to what extent the basic plasma components can be accounted for. We also provide a number of examples, giving an bird's eye overview of the current state of the field.

\section{Summary graphic}

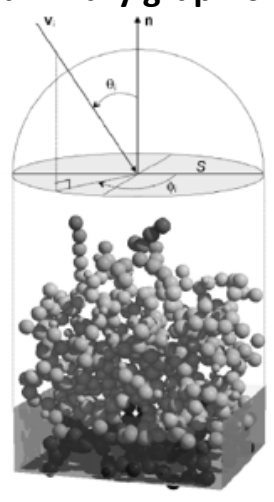

\section{TOC graphic}

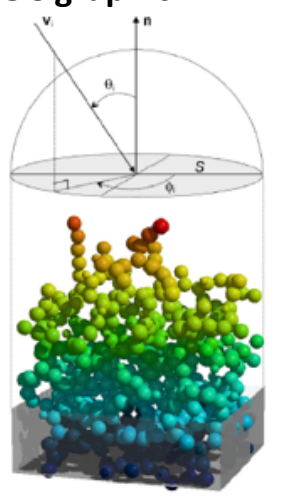

